\title{
Application of aptamers in medicinal plants
}

\section{Opinion}

From centuries ago, medicinal plants have been used for treatment of passions. Despite considerable improvement and development of synthetic drugs in recent decades, the use of herbal drugs continues to expand quickly across the world for treatment of various health challenges. In most countries medicinal plants and bio drugs are inseparable from synthetic drugs and treatment. Today, economical and marketing aspects of medicinal plants are more considered by bio science companies. Therefore, medicinal plant producers should using high technologies in order to enter and permanent presence in global marketing. As the global use of bio drugs continues to grow and many more new derivatives are introduced into the market, public healthcare issues and concerns surrounding their safety will also increasing. In fact, General healthcare concerns and rigid legislation from governments to import of bio drugs needs analysis and suitable controls of production of medicinal plants. Analyzing of medicinal plants by ordinary methods need to send samples to a laboratory and this process usually is time consuming, expensive, less sensitive and need practiced operator. But, researchers and producers are looking for monitoring farms and processing of medicinal plants using aptamerbased systems as a low cost, fast, sensitive and authentic technique.

Aptamers are short oligonucleotides, such as single-strand DNA (ssDNA) and RNA that due to their specific 3-D structures can bind to the targets with specificity and high affinity. Aptamers are selected by iterative cycles of affinity purification and amplification from a random oligonucleotide library which called Systematic Evolution of Ligands by Exponential enrichment (SELEX). SELEX was first reported by Gold's team in 1990, and due to development of this technique, which is a basic technique for production of aptamers, many aptamers can be select in vitro for various analyts, from cells to tiny biomolecules and bio drugs. Based on binding affinity, aptamers are similar to monoclonal antibody but provide extended applications. Aptamers are known as a good replacement for antibodies, because these molecules overcome the defect of antibodies. Due to the fully controlled synthesis, selective binding capability, object versatility, chemical and thermal stability, aptamers have become the main part of the research in all fields from nanotechnology and biotechnology to materials science. Aptamers have been applied as a bio-material in many investigations such as: a therapeutic and diagnostic tool, drug delivery systems, development of new drugs, and etc. Although researches and applications of aptamers are mainly focusing on various diseases, such as cancer and viral infection, there application in the medicinal plant and bio drug is in the beginning and needs more attentions. Potential application of aptamers in the medicinal plant industry can be summarized as:

\section{Biosensing using aptasensors}

A biosensor that is based on aptamers as a receptor is called an aptasensor. Aptasensors can be fabricated by a variety of methodologies, including optical biosensors, electrochemical biosensors, and colorimetric biosensors. An electrochemical aptasensor is an attractive devise, because it has high sensitivity, compatibility with novel nano-fabrication technologies, low cost

\author{
Volume 2 Issue 4 - 2017
}

\author{
Haidar Saify Nabiabad, Massoume Amini \\ Department of Medicinal Plant Production, Nahavand University, \\ Nahavand, Iran
}

Correspondence: Haidar Saify Nabiabad, Department of Medicinal Plant Production, Nahavand University, Nahavand, Iran, Tell +989183773206, Email homan_saify@yahoo.com

Received: May 05, 2017 | Published: December 29, 2017

and inherent miniaturization. Therefore, diverse electrochemical aptasensors have been designed using several techniques, including potentiometry EIS and DPV. To enhance the sensitivity of aptasensor, a nano particle uses as a material for immobilizing on the surface of the electrode. In optical aptasensors, aptamers are used as bio-probes based on the incorporation of a nanoparticle or a fluorophore. For fluorescence detection, the simplest model is labeling the aptamers with both a fluorophore and a quencher. In colorimetric detection technique, materials such as polymers and Au nanoparticles that cause color changes can be used as reagents. Single strand DNA (ssDNA) with highly negative charge, which is separated from the aptamer through interaction between the aptamer and the analyte, is fixed against aggregation and a color change occurs in association with this event.

\section{Monitoring of cultivation environment of medicinal plant}

Monitoring of cultivation environment of medicinal plant includes: detection of pathogens, measurement of heavy metals, allergens and toxins. Pathogens are main agents that limiting crop production and increasing economic losses. Detection of pathogens is important as the first step to manage a plant disease. It seems that aptasensor are promising tools for sensitive, fast and accurate detection of pathogens. Moreover, the determination of trace levels of toxic heavy metals, mycotoxins, pesticides and allergens in medicinal plant and bio drugs has become very important, because the consumers and health care organizations are vigilant and sensitive to this type of pollutants. Therefore, development of authentic analytical aptasensor is critical for ensuring fast and sensitive determination of bio drug contamination. A DNA aptamer was developed to detect ochratoxin A, a mycotoxin in plant product, with high specificity. An electrochemical gold nanoparticle based aptasensor for detection of endotoxins from crude biological liquor was developed by Kim et al. The aptasensor showed excellent sensitivity and selectivity with a detection range of 0.01 to $1 \mathrm{ngmL}^{-1}$. Moreover, there have been an increasing number of cases reporting severe allergic reactions to Lupin of herb seeds. To meet this challenge and detect Lupin allergen levels in herb product a DNA aptamer based colorimetric sensing system was developed by Nadal et al. [2012]. Endocrine disrupting compounds (EDC) form a major class of pollutants which cause s severe health hazard by disrupting normal endocrine functions among human and aquatic organisms. $17 \beta$-estradiol is one such compound which has deleterious effects 
on the male reproductive system. Recently, Yildrim et al. reported a highly selective and rapid fluorescence based DNA aptasensor for detection of low levels of $17 \beta$-estradiol in samples

\section{Identification of specific secondary metabolites}

Synthetic of secondary metabolites with pharmaceutics and economic value is expensive and sometimes impossible, so that the production of these bio drugs is much more economical and favorable than the synthetic types. Therefore, finding medicinal plant sources that contain acceptable quality and quantity of bio drugs are necessary. Although, methods such as GC, HPLC, and GC/MS are used for detection, but their application is time consuming and expensive. Therefore, aptasensor is recommended strongly for determination antioxidant capacity of medicinal plant extracts, and quantification valuable medicinal plant derivatives and secondary metabolite such as Vincristine, Vinblastine, and Taxol. In a study we fabricated an aptasensor for determination of Vinblastine (VLB) based on the covalent immobilization of a specific aptamer of VBL on a single walled carbon nanotube (SWCNTs) which were electrochemically deposited onto a GCE surface. In order to characterize the individual steps involved in the fabrication of the SWCNTs/GCE and VLBaptamer interaction at the electrode surface, the cyclic voltammetry and impedance spectroscopic techniques were used. A linear relationship between the electron-transfer resistance and VLB concentration was found in the range of 0.2 to $80.0 \mathrm{nmolL}^{-1}$ with a detection limit of $10 \times 10^{-2} \mathrm{nmolL}^{-1}$. Results show that the fabricated aptasensor has a wide linear range, high sensitivity, acceptable stability, and good selectivity and reproducibility. Thus, the proposed aptasensor and its fabrication procedure could appropriately determine VBL in medicinal plants such as Vinca minor L.

\section{Bio drug delivery systems}

Drug delivery system refers to methodologies, formulations and systems for transporting a pharmaceutical molecules into a specific tissue or part of body as needed to safely and effectively achieve its desired therapeutic effect. Drug delivery at slow delivery, targeted delivery, and controlled rate are very attractive approaches and have been investigated. For bio drug delivery, aptamers that bind to specific internal cell surface receptors are good candidate to deliver drugs into cells. In this direction production of specific aptamers for bio drugs, such as taxol, and integrates with specific aptamer of cancer cells will be promising in cancer therapy and other disease. Alibolandim, et al.[2015] reported doxorubicin (DOX)-encapsulating NPs formulated with PEG-PLGA nanopolymersome, wherein the surface was functionalized with EpCAM aptamer. The DOX-loaded aptamer conjugated nanopolymersomes could selectively bind with EpCAMpositive tumor cells (MCF-7), resulting in significantly greater cell toxicity than by non-targeted nanopolymersomes. Signore reported a novel oligonucleotide chimera, composed of a RNA aptamer and a DNA decoy. In this system, the RNA aptamer was used to target transferrin receptor (TfR), while DOX and nuclear factor $\mathrm{\kappa B}$ decoy oligonucleotide that inhibited the cell-survival factor were used as co-drugs. The aptamer motif promoted selective delivery of drugs to pancreatic tumor cells, and DOX exhibited $100 \%$ release under low $\mathrm{pH}$ value $(\mathrm{pH}=5.5)$, the co-delivery of a $\mathrm{\kappa B}$ decoy oligonucleotide greatly enhanced the DOX cytotoxicity to targeted pancreatic tumor cells due to the inhibition of constitutive NF- $\mathrm{KB}$ activity.

\section{Purification of bio drugs using aptamer affinity chromatography}

Immune-affinity Purification that relies on the interaction between an antibody and antigen is a common technique for purification of biomolecules. Specific-designed aptamer for a metabolite that should be purified and application in chromatography has many advantages over the use of an antibody, including a excellent specificity and affinity to the target, a smaller size, easier immobilization and modification, higher stability and reproducibility. In a study by Saify Nabiaba a specific aptamers were designed for tissue type plasminogen activator (tPA) using SELEX method. Multi wall carbon nanotubes were functionalized with selected aptamers, packed in a column, and used for purification of tPA from tobacco hairy roots. The results demonstrated that selected aptamer having KD values of $0.320 \mathrm{nM}$ and IC50 of $28.9 \mathrm{nM}$ possessed good affinity to tPA, and the chimeric-tPA was properly purified by aptamer-chromatography.

\section{Conclusion}

Aptamers, as chemical antibody, due to their high specificity, binding affinity, flexibility of configuration and production costs have attracted much attention in biomedical fields. In this Opinion, we summarized the recent progresses and potential application of aptamer-based systems in medicinal plant industry. Aptamers will emerging as a powerful player in the herbal drug production including but not limited to sensing, monitoring, drug delivery and bio drug purification. It should be noted, selection of aptamers with the highest selectivity and sensitivity for a range of targets, especially the secondary metabolites with pharmaceutical importance, will be of interest to future researchers and industrials specialized in biotechnologies. With the wide spread use of aptamer-functionalized systems, more and more scientists and pharmaceutical companies are realizing their promising application prospects and we are confident that aptamer-based products will begin entering markets in the near future.

\section{Acknowledgements}

None.

\section{Conflict of interest}

The author declares no conflict of interest. 